\title{
Patient-reported outcomes during repetitive oxaliplatin-based pressurized intraperitoneal aerosol chemotherapy for isolated unresectable colorectal peritoneal metastases in a multicenter, single-arm, phase 2 trial (CRC-PIPAC)
}

\author{
Robin J. Lurvink ${ }^{1,2}$ - Koen P. Rovers ${ }^{1} \cdot$ Emma C. E. Wassenaar $^{3} \cdot$ Checca Bakkers $^{1}$ - Jacobus W. A. Burger ${ }^{1}$. \\ Geert-Jan M. Creemers ${ }^{4} \cdot$ Maartje Los ${ }^{5}$. Floortje Mols ${ }^{2,6} \cdot$ Marinus J. Wiezer $^{3} \cdot$ Simon W. Nienhuijs ${ }^{1}$. \\ Djamila Boerma ${ }^{3} \cdot$ Ignace H. J. T. de Hingh ${ }^{1,2,7}$
}

Received: 26 April 2021 / Accepted: 17 October 2021 / Published online: 10 November 2021

(c) The Author(s) 2021

\begin{abstract}
Background CRC-PIPAC prospectively assessed repetitive oxaliplatin-based pressurized intraperitoneal aerosol chemotherapy (PIPAC-OX) as a palliative monotherapy (i.e., without concomitant systemic therapy in between subsequent procedures) for unresectable colorectal peritoneal metastases (CPM). The present study explored patient-reported outcomes (PROs) during trial treatment.

Methods In this single-arm phase 2 trial in two tertiary centers, patients with isolated unresectable CPM received 6-weekly PIPAC-OX $\left(92 \mathrm{mg} / \mathrm{m}^{2}\right)$. PROs (calculated from EQ-5D-5L, and EORTC QLQ-C30 and QLQ-CR29) were compared between baseline and 1 and 4 weeks after the first three procedures using linear mixed modeling with determination of clinical relevance (Cohen's $D \geq 0.50$ ) of statistically significant differences.

Results Twenty patients underwent 59 procedures (median 3 [range 1-6]). Several PROs solely worsened 1 week after the first procedure (index value $-0.10, p<0.001$; physical functioning $-20, p<0.001$; role functioning $-27, p<0.001$; social functioning $-18, p<0.001$; C30 summary score $-16, p<0.001$; appetite loss $+15, p=0.007$; diarrhea $+15, p=0.002$; urinary frequency $+13, p=0.004$; flatulence $+13, p=0.001$ ). These PROs returned to baseline at subsequent time points. Other PROs worsened 1 week after the first procedure (fatigue $+23, p<0.001$; pain $+29, p<0.001$; abdominal pain +32 , $p<0.001$ ), second procedure (fatigue $+20, p<0.001$; pain $+21, p<0.001$; abdominal pain $+20, p=0.002$ ), and third procedure (pain $+22, p<0.001$; abdominal pain $+22, p=0.002$ ). Except for appetite loss, all changes were clinically relevant. All analyzed PROs returned to baseline 4 weeks after the third procedure.

Conclusions Patients receiving repetitive PIPAC-OX monotherapy for unresectable CPM had clinically relevant but reversible worsening of several PROs, mainly 1 week after the first procedure.
\end{abstract}

Trial registration Clinicaltrials.gov: NCT03246321; Netherlands trial register: NL6426.

Keywords Colorectal neoplasms $\cdot$ Peritoneal neoplasms $\cdot$ Antineoplastic agents $\cdot$ Oxaliplatin $\cdot$ Patient-reported outcome measures · Quality of life

The peritoneum is a common and often lethal metastatic site of colorectal cancer $[1,2]$. The majority of patients with colorectal peritoneal metastases (CPM) are treated

Robin J. Lurvink and Koen P. Rovers are equally contributing first authors.

Ignace H. J. T. de Hingh

ignace.d.hingh@catharinaziekenhuis.nl

Extended author information available on the last page of the article with palliative intent $[3,4]$. Theoretically, intraperitoneal chemotherapy could be an interesting palliative treatment option in patients with isolated peritoneal metastases, as it may achieve high locoregional efficacy with low systemic toxicity [5]. However, its use appears to be limited due to an inhomogeneous intraperitoneal distribution, dose-limiting local toxicity, and poor tumor penetration $[6,7]$. To overcome these limitations, a laparoscopic method for the repetitive delivery of low-dose intraperitoneal chemotherapy as a 
pressurized aerosol (i.e., pressurized intraperitoneal aerosol chemotherapy [PIPAC]) has been developed. PIPAC claims to result in enhanced tumor penetration, homogeneous intraperitoneal distribution, and low systemic toxicity [8-11]. The intriguing concept and promising preliminary results have led to the adoption of PIPAC as a palliative treatment option for isolated unresectable CPM in a rapidly increasing number of hospitals worldwide [12]. In these hospitals, these patients regularly receive oxaliplatin-based PIPAC (PIPAC$\mathrm{OX}$ ) with or without concomitant palliative systemic therapy in a dose of $90-92 \mathrm{mg} / \mathrm{m}^{2}$ every 4 to 8 weeks [13]. Despite its increasing use, repetitive PIPAC-OX has never been prospectively investigated as a palliative monotherapy (i.e., without palliative systemic therapy in between subsequent procedures) for isolated unresectable CPM in clinical trials. As a first step to address this evidence gap, the CRC-PIPAC trial primarily aimed to assess the feasibility, safety, preliminary efficacy, survival outcomes, and patient-reported outcomes (PROs) of repetitive PIPAC-OX monotherapy for isolated unresectable CPM $[14,15]$. The aim of the present study was to explore PROs during trial treatment.

\section{Materials and methods}

\section{Trial design}

CRC-PIPAC was a single-arm phase 2 trial conducted in two Dutch tertiary centers for the surgical treatment of CPM. The trial was approved by a central ethics committee (MEC-U, Nieuwegein, Netherlands, R17.038) and the institutional review boards of both trial centers. The trial is registered (Clinicaltrials.gov: NCT03246321), and the protocol has been previously published [14].

\section{Patients}

The protocol includes a detailed description of the eligibility criteria [14]. Briefly, eligible patients were adults with a World Health Organization performance status of $0-1$, pathologically proven isolated unresectable peritoneal metastases of a colorectal or appendiceal carcinoma (or high-grade appendiceal mucinous neoplasm), adequate organ functions, no symptoms of gastrointestinal obstruction, no contraindications for laparoscopy or the planned chemotherapy, and no previous PIPAC, in any line of palliative treatment. Patients were informed about the potential consequences of discontinuing or postponing standard palliative treatment, were discussed in a multidisciplinary team prior to enrollment, and gave written informed consent.

\section{Procedures}

\section{PIPAC-OX}

The protocol comprises a detailed description of the procedure [14], which is based on internationally used protocols [13]. Patients underwent 6-weekly PIPAC-OX (92 $\mathrm{mg} / \mathrm{m}^{2}$, maximum $184 \mathrm{mg}$ ) with a simultaneous intravenous bolus 5 -fluorouracil $\left(400 \mathrm{mg} / \mathrm{m}^{2}\right)$ and leucovorin $\left(20 \mathrm{mg} / \mathrm{m}^{2}\right)[16,17]$ and electrostatic aerosol precipitation (i.e., ePIPAC-OX) [18, 19]. Electrostatic precipitation was started directly after complete injection of the aerosol, after which the total procedure was maintained for $25 \mathrm{~min}$. If possible, patients were discharged on the first postoperative day. No concomitant palliative systemic therapy was given in between subsequent procedures (i.e., ePIPAC-OX monotherapy).

\section{Evaluations}

Four weeks after each procedure, patients were clinically, biochemically, and radiologically evaluated. Trial treatment was stopped in case of radiological progression according to the response evaluation criteria in solid tumors (RECIST) [20]. In case of RECIST non-evaluable or stable disease (or response), the decision to continue or stop trial treatment was made by shared decision based on previous treatment, remaining treatment options, clinical parameters (e.g., toxicity, symptoms), biochemical parameters (e.g., tumor markers), macroscopic parameters (e.g., ascites volume), and secondary radiological parameters (e.g., radiological peritoneal cancer index). If trial treatment was stopped, patients received off-protocol palliative treatment.

\section{PROs}

Patients were asked to complete three questionnaires (EuroQoL EQ-5D-5L [21], EORTC QLQ-C30 [22], and EORTC QLQ-CR29 [23] at baseline and 1 and 4 weeks after each procedure. As the trial's aim was to assess PROs during trial treatment, patients were not asked to complete questionnaires after discontinuation of trial treatment (e.g., due to disease progression) or during follow-up. At patient's preference, questionnaires were sent on paper or electronically using certified software (Research Manager, Deventer, the Netherlands). Table 1 presents the PRO categories of each questionnaire. Scores for each category were calculated according to the manuals of EuroQol and EORTC [24-26]. Scores range from 0 to 100 except for 
Table 1 PROs of each questionnaire

\begin{tabular}{|c|c|c|}
\hline Questionnaire & Function scales ${ }^{\mathrm{a}}$ & Symptom scales ${ }^{b}$ \\
\hline EQ-5D-5L & $\begin{array}{l}\text { - Visual analog scale } \\
\text { - Index value }\end{array}$ & \\
\hline EORTC QLQ-C30 & $\begin{array}{l}\text { - Global health status } \\
\text { - Physical function- } \\
\text { ing } \\
\text { - Role functioning } \\
\text { - Emotional func- } \\
\text { tioning } \\
\text { - Cognitive function- } \\
\text { ing } \\
\text { - Social functioning } \\
\text { - C30 summary score }\end{array}$ & $\begin{array}{l}\text { - Fatigue } \\
\text { - Nausea/vomiting } \\
\text { - Pain } \\
\text { - Dyspnea } \\
\text { - Insomnia } \\
\text { - Appetite loss } \\
\text { - Constipation } \\
\text { - Diarrhea } \\
\text { - Financial difficulties }\end{array}$ \\
\hline EORTC QLQ-CR29 & $\begin{array}{l}\text { - Anxiety } \\
\text { - Weight } \\
\text { - Body image } \\
\text { - Sexual interest } \\
\text { (males) } \\
\text { - Sexual interest } \\
\quad \text { (females) }\end{array}$ & $\begin{array}{l}\text { - Urinary frequency } \\
\text { - Urinary incontinence } \\
\text { - Dysuria } \\
\text { - Abdominal pain } \\
\text { - Buttock pain } \\
\text { - Bloating } \\
\text { - Blood/mucus in stool } \\
\text { - Dry mouth } \\
\text { - Hair loss } \\
\text { - Taste } \\
\text { - Flatulence } \\
\text { - Fecal incontinence } \\
\text { - Sore skin } \\
\text { - Stool frequency } \\
\text { - Embarrassment } \\
\text { - Stoma care problems } \\
\text { - Impotence (males) } \\
\text { - Dyspareunia } \\
\text { (females) }\end{array}$ \\
\hline
\end{tabular}

${ }^{\mathrm{a}}$ Lower scores indicate worse functioning

${ }^{\mathrm{b}}$ Higher scores indicate worse symptoms

the index value of EQ-5D-5L, which ranges from -0.329 to 1.00 according to the Dutch value set [27]. In general, PRO categories can be divided in function scales (with lower scores indicating worse functioning) and symptom scales (with higher scores indicating worse symptoms) (Table 1).

\section{Statistical analysis}

Since no data were available to guide a sample size calculation, the investigators and the ethics committee agreed upon a sample size of 20 patients undergoing an estimated number of 60 procedures as sufficient numbers to explore the safety and feasibility of the intervention in the CRCPIPAC trial. As PRO assessment of the present study was explorative, no a priori hypothesis for PRO analyses was formulated. Consequently, the scores of all PRO categories up to 4 weeks after the third procedure were included in the analyses. Analyses were performed two sided using IBM SPSS Statistics (version 25.0, Armonk, NY, United States). To account for multiple testing, Bonferroni corrections were applied for each PRO category. Hence, $p<0.0083$ was considered statistically significant (i.e., $\alpha=0.05$ divided by 6 comparisons per PRO category). For each PRO category, changes in scores between baseline and subsequent time points were presented as a mean difference (MD) and analyzed using linear mixed modeling. Pairwise deletion was used in case of missing values. All PRO categories with a statistically significant difference in scores between baseline and at least one subsequent time point were presented. For all PRO categories, Cohen's d (CD) effect sizes were calculated to determine the clinical relevance of each statistically significant difference, with a CD of $\geq 0.5$ being considered clinically relevant [28]. Furthermore, patient-based clinical thresholds were used to determine whether deteriorations or improvements of PROs were major, moderate, or minor (EORTC QLQ-C30 [29], EORTC QLQ-CR29) [30] and to determine whether a change in a PRO exceeded a minimally important difference (EuroQol EQ-5D-5L) [31]. Since mean scores were used to present changes over time and determine effect sizes, all scores were presented as a mean (standard deviation) regardless of distribution.

\section{Results}

Between October 10, 2017, and September 24, 2018, 43 patients were screened for eligibility, of whom 20 were included in the PRO analyses (Fig. 1). Baseline characteristics are presented in Table 2. Between October 30, 2017, and April 24, 2019, these 20 patients underwent 59 (median 3 [range 1-6]) procedures. Figure 1 presents the patient pathway and questionnaire response rates at each time point, including reasons for non-response and discontinuation of trial treatment.

Table 3 presents the mean scores of all PRO categories at each time point. Online Appendix A presents the linear mixed modeling analyses of the 12 PRO categories with a statistically significant difference. The scores of all other 29 PRO categories did not significantly change during trial treatment (linear mixed modeling analyses presented in Online Appendix B).

\section{Changing function scales}

\section{Index value}

Compared to baseline, index value worsened 1 week after the first procedure (MD -0.10 [95\% confidence interval: -0.16 to -0.05 ], $p<0.001, \mathrm{CD} 0.76$, exceeds minimally important difference) and returned to baseline at subsequent time points (Fig. 2A). 


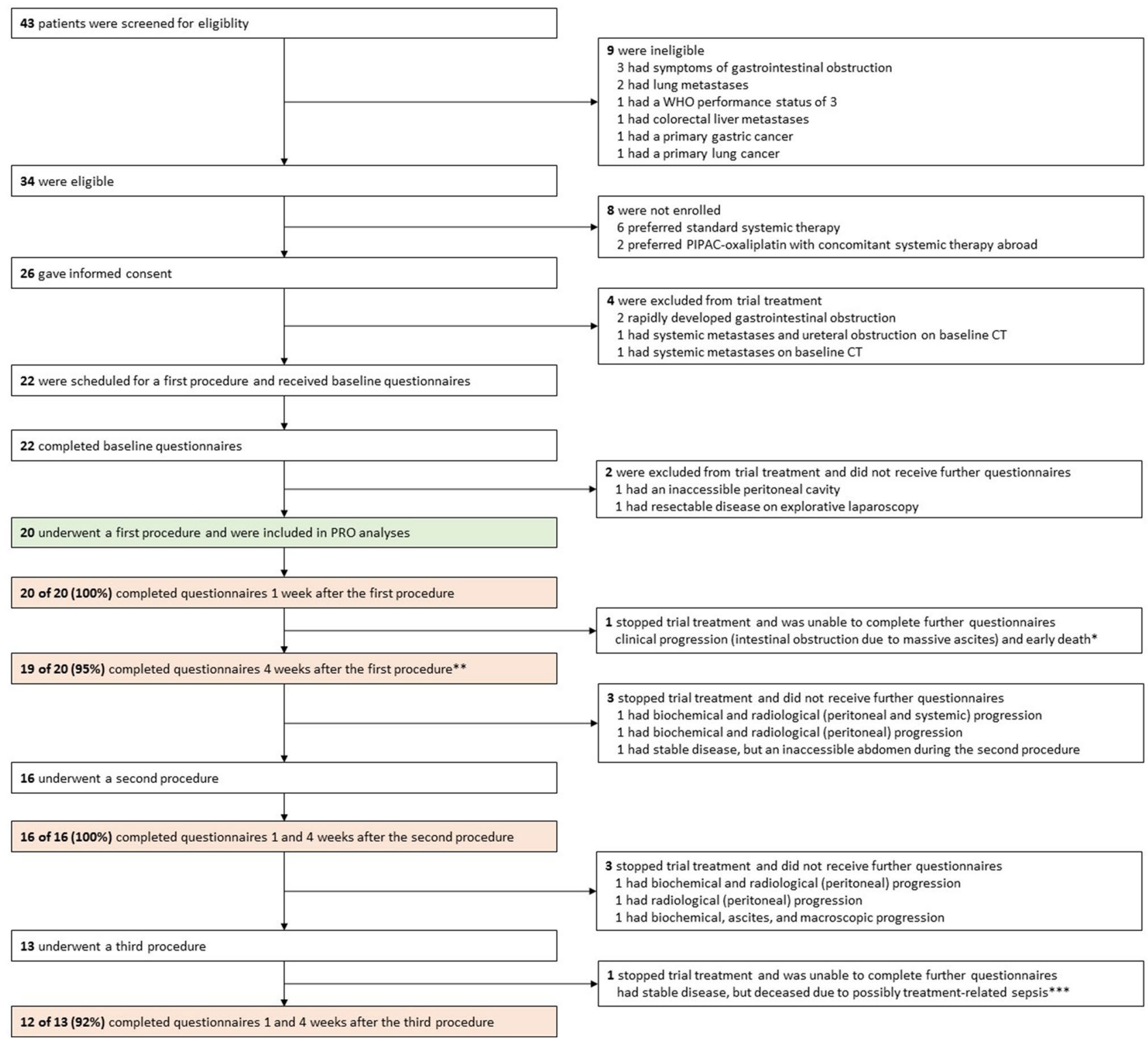

Fig. 1 Patient pathway and questionnaire response rates (including reasons for non-response) at each time point. $W H O$ world health organization; *deceased 2 weeks postoperatively; **one patient com-

\section{Physical functioning}

Compared to baseline, physical functioning worsened 1 week after the first procedure (MD - 20 [95\% CI - 27 to $-12], p<0.001, \mathrm{CD} 1.03$, major deterioration) and returned to baseline at subsequent time points (Fig. 2B).

\section{Role functioning}

Compared to baseline, role functioning worsened 1 week after the first procedure (MD -27 [95\% CI -39 to -15$]$, pleted EQ-5D-5L and EORTC QLQ-CR29, but accidentally forgot to fill in most questions of EORTC QLQ-CR30; ***deceased five days postoperatively

$p<0.001, \mathrm{CD} 0.87$, major deterioration) and returned to baseline at subsequent time points (Fig. 2C).

\section{Social functioning}

Compared to baseline, social functioning worsened 1 week after the first procedure (MD - 18 [95\% CI -28 to -8$]$, $p<0.001, \mathrm{CD} 0.71$, moderate deterioration) and returned to baseline at subsequent time points (Fig. 2D). 
Table 2 Baseline characteristics

Sex
Male
Female
Years of age at enrollment, median (range)
WHO performance status

20

$12(60 \%)$

$8(40 \%)$

$64(41-78)$

WHO performance status

20

$8(40 \%)$

$10(50 \%)$

$2(10 \%)^{\mathrm{a}}$

$\geq 2^{\mathrm{a}}$

20

$7(35 \%)$

$6(30 \%)$

$7(35 \%)$

Appendix

13

$4(31 \%)$

$5(38 \%)$

$4(31 \%)$

7

$1(14 \%)$

$4(57 \%)$

$2(29 \%)^{\mathrm{b}}$

20

$6(30 \%)$

$5(25 \%)$

$9(45 \%)$

20

$15(75 \%)$

$5(25 \%)$

$4(1-32)$

20

$11(55 \%)$

$1(5 \%)$

$8(40 \%)$

15

$6(40 \%)^{c}$

$8(53 \%)$

$1(7 \%)$

5

$2(40 \%)^{\mathrm{c}}$

$2(40 \%)^{\mathrm{c}}$

$1(20 \%)$

$11^{\mathrm{d}}$

$8(73 \%)$

$3(27 \%)^{\mathrm{d}}$

20

$13(65 \%)$

$6(30 \%)$

$1(5 \%)$

20

$13(65 \%)$

$3(15 \%)$

$4(20 \%)$

$260(60-6000)^{\mathrm{e}}$ 
Table 2 (continued)

$\begin{array}{ll}\text { Radiological peritoneal cancer index at baseline radiology, median (range) } & 31(11-39) \\ \text { Macroscopic peritoneal cancer index during first laparoscopy, median (range) } & 29(17-39)\end{array}$

WHO world health organization

${ }^{\mathrm{a}}$ Both deteriorated between enrollment (WHO 1) and the first procedure (one WHO 2, one WHO 3) due to increasing ascites

${ }^{b}$ Pre-trial biopsies classified as high-grade appendiceal mucinous neoplasm, but biopsies during the trial revealed low-grade appendiceal mucinous neoplasm

${ }^{c}$ Either refused—or preferred enrollment rather than starting with—first- or second-line palliative systemic therapy

${ }^{\mathrm{d}}$ One had a wait-and-see strategy

${ }^{\mathrm{e}}$ In those with $\geq 50 \mathrm{ml}$

\section{C30 summary score}

Compared to baseline, C30 summary score worsened 1 week after the first procedure (MD - 16 [95\% CI -20 to -9], $p<0.001, \mathrm{CD} 1.07$, moderate deterioration) and returned to baseline at subsequent time points (Fig. 2E).

\section{Changing symptom scales}

\section{Fatigue}

Compared to baseline, fatigue worsened 1 week after the first procedure (MD + 23 [95\% CI 14-33], $p<0.001, \mathrm{CD}$ 0.98 , major deterioration), returned to baseline 4 weeks after the first procedure $(p=0.13)$, worsened 1 week after the second procedure (MD+20 [95\% CI 9-30], $p<0.001, \mathrm{CD} 0.83$, major deterioration), and returned to baseline at subsequent time points (Fig. 3A).

\section{Pain}

Compared to baseline, pain was worse 1 week after the first procedure (MD + 29 [95\% CI 19-40], $p<0.001$, CD 1.49, major deterioration), second procedure (MD +21 [95\% CI 10-33], $p<0.001, \mathrm{CD} 0.95$, major deterioration), and third procedure (MD + 22, [95\% CI 11-36], $p<0.001, \mathrm{CD} 0.95$, major deterioration), and was comparable to baseline 4 weeks after each procedure (Fig. 3B).

\section{Appetite loss}

Compared to baseline, appetite loss worsened 1 week after the first procedure (MD+15 [95\% CI 4-26], $p=0.007, \mathrm{CD}$ 0.43 , moderate deterioration) and returned to baseline at subsequent time points (Fig. 3C).

\section{Diarrhea}

Compared to baseline, diarrhea worsened 1 week after the first procedure (MD+ 15 [95\% CI 5-25], $p=0.002, \mathrm{CD} 0.65$, minor deterioration) and returned to baseline at subsequent time points (Fig. 3D).

\section{Urinary frequency}

Compared to baseline, urinary frequency worsened 1 week after the first procedure (MD+13 [95\% CI 4-22], $p=0.004$, CD 0.74 , moderate deterioration) and returned to baseline at subsequent time points (Fig. 3E).

\section{Abdominal pain}

Compared to baseline, abdominal pain was worse 1 week after the first procedure (MD+32 [95\% CI 20-43], $p<0.001, \mathrm{CD} 1.58$, major deterioration), 4 weeks after the first procedure (MD + 19 [95\% CI 7-31], $p=0.003, \mathrm{CD}$ 0.89 , moderate deterioration), 1 week after the second procedure (MD + 20 [95\% CI 7-33], $p=0.002, \mathrm{CD} 0.86$, moderate deterioration), and 1 week after the third procedure $(\mathrm{MD}+22$ [95\% CI 9-36], $p=0.002, \mathrm{CD} 1.03$, major deterioration), and was comparable to baseline 4 weeks after the second and third procedures (Fig. 3F).

\section{Flatulence}

Compared to baseline, flatulence worsened 1 week after the first procedure (MD+13 [95\% CI 6-21], $p=0.001, \mathrm{CD} 0.56$, moderate deterioration) and returned to baseline at subsequent time points (Fig. 3G).

\section{Discussion}

The present study showed that patients with isolated unresectable CPM receiving repetitive ePIPAC-OX monotherapy had worsening of several general (index value, physical functioning, role functioning, social functioning, C30 summary score, fatigue) and more specific (pain, appetite loss, diarrhea, urinary frequency, abdominal pain, flatulence) PROs during trial treatment. The majority of these PROs worsened 
Table 3 Mean scores with standard deviations of all PROs at each time point

EuroQoL EQ-5D-5L

\begin{tabular}{|c|c|c|c|c|c|c|c|}
\hline PRO & Baseline & $\begin{array}{l}1 \text { week after } \\
1 \text { st procedure }\end{array}$ & $\begin{array}{l}4 \text { weeks after } \\
1 \text { st procedure }\end{array}$ & $\begin{array}{l}1 \text { week after } \\
2 \text { nd procedure }\end{array}$ & $\begin{array}{l}4 \text { weeks } \\
\text { after } 2 \text { nd } \\
\text { procedure }\end{array}$ & $\begin{array}{l}1 \text { week } \\
\text { after 3rd } \\
\text { procedure }\end{array}$ & $\begin{array}{l}4 \text { weeks } \\
\text { after 3rd procedure }\end{array}$ \\
\hline Visual analog scale & $62 \pm 28$ & $54 \pm 29$ & $64 \pm 24$ & $64 \pm 21$ & $59 \pm 32$ & $66 \pm 23$ & $63 \pm 30$ \\
\hline Index value & $0.84 \pm 0.11$ & $0.74 \pm 0.15$ & $0.82 \pm 0.14$ & $0.81 \pm 0.12$ & $0.83 \pm 0.14$ & $0.80 \pm 0.18$ & $0.85 \pm 0.14$ \\
\hline \multicolumn{8}{|l|}{ EORTC QLQ-C30 } \\
\hline PRO & Baseline & $\begin{array}{l}1 \text { week after } \\
1 \text { st procedure }\end{array}$ & $\begin{array}{l}4 \text { weeks after } \\
1 \text { st procedure }\end{array}$ & $\begin{array}{l}1 \text { week after } \\
2 \text { nd procedure }\end{array}$ & $\begin{array}{l}4 \text { weeks } \\
\text { after } 2 \text { nd } \\
\text { procedure }\end{array}$ & $\begin{array}{l}1 \text { week } \\
\text { after } 3 \text { rd } \\
\text { procedure }\end{array}$ & $\begin{array}{l}4 \text { weeks } \\
\text { after 3rd procedure }\end{array}$ \\
\hline Global health status & $65 \pm 29$ & $57 \pm 21$ & $67 \pm 25$ & $60 \pm 25$ & $65 \pm 24$ & $63 \pm 22$ & $67 \pm 24$ \\
\hline Physical functioning & $87 \pm 15$ & $67 \pm 23$ & $81 \pm 20$ & $76 \pm 18$ & $81 \pm 20$ & $78 \pm 19$ & $86 \pm 20$ \\
\hline Role functioning & $69 \pm 30$ & $42 \pm 32$ & $69 \pm 28$ & $53 \pm 31$ & $64 \pm 39$ & $56 \pm 30$ & $76 \pm 29$ \\
\hline Emotional functioning & $79 \pm 23$ & $78 \pm 23$ & $81 \pm 18$ & $74 \pm 20$ & $78 \pm 23$ & $78 \pm 22$ & $76 \pm 23$ \\
\hline Cognitive functioning & $88 \pm 17$ & $82 \pm 17$ & $91 \pm 12$ & $87 \pm 16$ & $96 \pm 10$ & $90 \pm 13$ & $90 \pm 17$ \\
\hline Social functioning & $80 \pm 27$ & $62 \pm 24$ & $79 \pm 23$ & $79 \pm 25$ & $81 \pm 25$ & $76 \pm 32$ & $85 \pm 30$ \\
\hline Fatigue & $30 \pm 23$ & $53 \pm 24$ & $37 \pm 25$ & $50 \pm 25$ & $35 \pm 30$ & $43 \pm 28$ & $33 \pm 28$ \\
\hline Nausea/vomiting & $13 \pm 25$ & $19 \pm 27$ & $8 \pm 13$ & $11 \pm 19$ & $7 \pm 12$ & $14 \pm 17$ & $6 \pm 15$ \\
\hline Pain & $21 \pm 20$ & $50 \pm 19$ & $29 \pm 18$ & $42 \pm 24$ & $29 \pm 27$ & $43 \pm 26$ & $25 \pm 23$ \\
\hline Dyspnea & $12 \pm 20$ & $13 \pm 20$ & $9 \pm 19$ & $15 \pm 24$ & $6 \pm 13$ & $8 \pm 21$ & $6 \pm 19$ \\
\hline Insomnia & $17 \pm 23$ & $23 \pm 31$ & $24 \pm 22$ & $31 \pm 28$ & $19 \pm 24$ & $17 \pm 17$ & $14 \pm 22$ \\
\hline Appetite loss & $25 \pm 37$ & $40 \pm 32$ & $24 \pm 36$ & $37 \pm 34$ & $27 \pm 33$ & $39 \pm 31$ & $25 \pm 32$ \\
\hline Constipation & $8 \pm 15$ & $22 \pm 27$ & $13 \pm 26$ & $12 \pm 21$ & $6 \pm 13$ & $11 \pm 22$ & $3 \pm 10$ \\
\hline Diarrhea & $12 \pm 20$ & $27 \pm 26$ & $19 \pm 21$ & $6 \pm 13$ & $10 \pm 16$ & $17 \pm 27$ & $14 \pm 17$ \\
\hline Financial difficulties & $3 \pm 15$ & $10 \pm 27$ & $2 \pm 8$ & $2 \pm 8$ & $0 \pm 0$ & $3 \pm 10$ & $3 \pm 10$ \\
\hline $\mathrm{C} 30$ summary score & $82 \pm 15$ & $66 \pm 15$ & $80 \pm 14$ & $75 \pm 16$ & $81 \pm 16$ & $76 \pm 16$ & $84 \pm 18$ \\
\hline
\end{tabular}

EORTC QLQ-CR29

\begin{tabular}{|c|c|c|c|c|c|c|c|}
\hline PRO & Baseline & $\begin{array}{l}1 \text { week after } \\
1 \text { st procedure }\end{array}$ & $\begin{array}{l}4 \text { weeks after } \\
1 \text { st procedure }\end{array}$ & $\begin{array}{l}1 \text { week after } \\
2 \text { nd procedure }\end{array}$ & $\begin{array}{l}4 \text { weeks } \\
\text { after } 2 \text { nd } \\
\text { procedure }\end{array}$ & $\begin{array}{l}1 \text { week } \\
\text { after } 3 \text { rd } \\
\text { procedure }\end{array}$ & $\begin{array}{l}4 \text { weeks } \\
\text { after 3rd procedure }\end{array}$ \\
\hline Urinary frequency & $12 \pm 17$ & $25 \pm 18$ & $24 \pm 20$ & $26 \pm 18$ & $21 \pm 22$ & $26 \pm 19$ & $19 \pm 16$ \\
\hline Urinary incontinence & $2 \pm 7$ & $0 \pm 0$ & $4 \pm 11$ & $4 \pm 11$ & $4 \pm 12$ & $3 \pm 10$ & $0 \pm 0$ \\
\hline Dysuria & $3 \pm 10$ & $7 \pm 14$ & $7 \pm 18$ & $6 \pm 13$ & $10 \pm 26$ & $6 \pm 13$ & $6 \pm 13$ \\
\hline Abdominal pain & $20 \pm 17$ & $52 \pm 23$ & $39 \pm 25$ & $40 \pm 28$ & $35 \pm 33$ & $42 \pm 25$ & $22 \pm 26$ \\
\hline Buttock pain & $7 \pm 14$ & $5 \pm 16$ & $11 \pm 19$ & $6 \pm 13$ & $6 \pm 13$ & $8 \pm 15$ & $3 \pm 10$ \\
\hline Bloating & $28 \pm 27$ & $35 \pm 23$ & $23 \pm 25$ & $33 \pm 30$ & $29 \pm 32$ & $28 \pm 31$ & $22 \pm 30$ \\
\hline Blood/mucus in stool & $0 \pm 0$ & $3 \pm 9$ & $3 \pm 6$ & $2 \pm 6$ & $3 \pm 7$ & $1 \pm 5$ & $0 \pm 0$ \\
\hline Dry mouth & $18 \pm 23$ & $27 \pm 32$ & $24 \pm 25$ & $25 \pm 23$ & $19 \pm 21$ & $22 \pm 22$ & $17 \pm 22$ \\
\hline Hair loss & $3 \pm 10$ & $5 \pm 12$ & $5 \pm 12$ & $4 \pm 11$ & $8 \pm 19$ & $8 \pm 21$ & $11 \pm 30$ \\
\hline Taste & $18 \pm 30$ & $22 \pm 31$ & $16 \pm 23$ & $27 \pm 30$ & $23 \pm 26$ & $19 \pm 26$ & $14 \pm 22$ \\
\hline Flatulence & $17 \pm 20$ & $30 \pm 26$ & $21 \pm 23$ & $15 \pm 17$ & $8 \pm 15$ & $14 \pm 17$ & $14 \pm 17$ \\
\hline Fecal incontinence & $5 \pm 16$ & $5 \pm 12$ & $4 \pm 11$ & $2 \pm 8$ & $2 \pm 8$ & $3 \pm 10$ & $0 \pm 0$ \\
\hline Sore skin & $7 \pm 14$ & $10 \pm 19$ & $11 \pm 19$ & $2 \pm 8$ & $4 \pm 11$ & $6 \pm 13$ & $6 \pm 19$ \\
\hline Stool frequency & $8 \pm 11$ & $15 \pm 23$ & $5 \pm 8$ & $7 \pm 10$ & $6 \pm 10$ & $7 \pm 15$ & $7 \pm 11$ \\
\hline Embarrassment & $10 \pm 19$ & $10 \pm 19$ & $7 \pm 14$ & $10 \pm 16$ & $4 \pm 11$ & $3 \pm 10$ & $3 \pm 10$ \\
\hline Stoma care problems & $8 \pm 17$ & $8 \pm 17$ & $0 \pm 0$ & $17 \pm 24$ & $33 \pm 47$ & $17 \pm 24$ & $17 \pm 24$ \\
\hline Impotence (males) & $3 \pm 10$ & $15 \pm 23$ & $12 \pm 17$ & $8 \pm 15$ & $8 \pm 15$ & $13 \pm 17$ & $4 \pm 12$ \\
\hline Dyspareunia (females) & $4 \pm 12$ & $0 \pm 0$ & $0 \pm 0$ & $0 \pm 0$ & $10 \pm 16$ & $11 \pm 19$ & $17 \pm 19$ \\
\hline Anxiety & $53 \pm 29$ & $62 \pm 33$ & $60 \pm 33$ & $56 \pm 32$ & $60 \pm 35$ & $56 \pm 30$ & $61 \pm 28$ \\
\hline Weight & $80 \pm 33$ & $77 \pm 33$ & $84 \pm 23$ & $83 \pm 24$ & $75 \pm 31$ & $75 \pm 35$ & $78 \pm 36$ \\
\hline
\end{tabular}


Table 3 (continued)

\begin{tabular}{|c|c|c|c|c|c|c|c|}
\hline \multicolumn{8}{|l|}{ EORTC QLQ-CR29 } \\
\hline PRO & Baseline & $\begin{array}{l}1 \text { week after } \\
1 \text { st procedure }\end{array}$ & $\begin{array}{l}4 \text { weeks after } \\
1 \text { st procedure }\end{array}$ & $\begin{array}{l}1 \text { week after } \\
2 \text { nd procedure }\end{array}$ & $\begin{array}{l}4 \text { weeks } \\
\text { after } 2 \text { nd } \\
\text { procedure }\end{array}$ & $\begin{array}{l}1 \text { week } \\
\text { after } 3 \text { rd } \\
\text { procedure }\end{array}$ & $\begin{array}{l}4 \text { weeks } \\
\text { after } 3 \text { rd procedure }\end{array}$ \\
\hline Body image & $87 \pm 17$ & $83 \pm 23$ & $90 \pm 14$ & $84 \pm 22$ & $85 \pm 21$ & $82 \pm 25$ & $81 \pm 23$ \\
\hline Sexual interest (males) & $31 \pm 30$ & $22 \pm 26$ & $25 \pm 29$ & $22 \pm 24$ & $19 \pm 24$ & $21 \pm 25$ & $25 \pm 30$ \\
\hline Sexual interest (females) & $8 \pm 15$ & $4 \pm 12$ & $10 \pm 16$ & $5 \pm 13$ & $10 \pm 16$ & $0 \pm 0$ & $8 \pm 17$ \\
\hline
\end{tabular}

$P R O$ patient-reported outcome
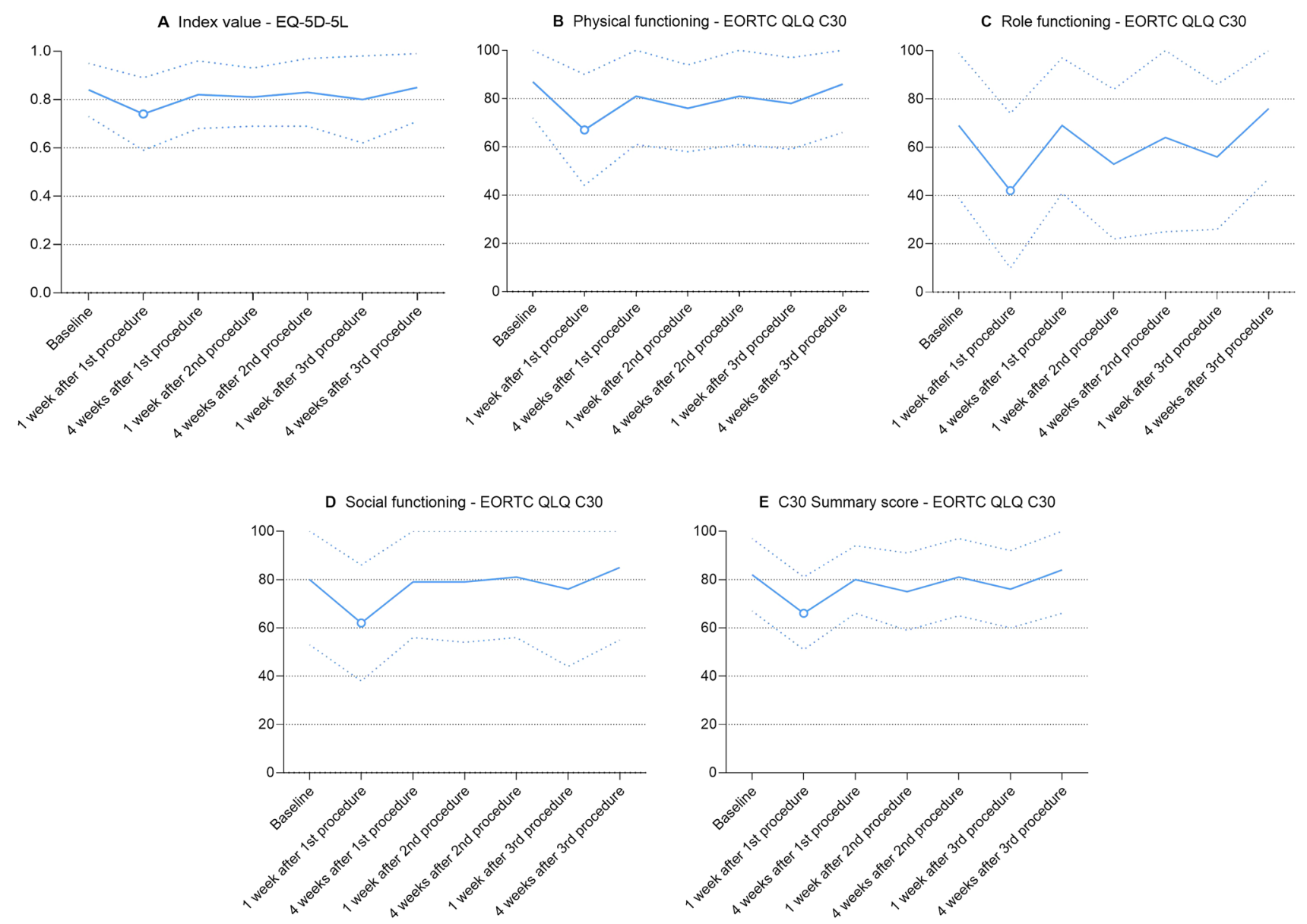

Fig. 2 All function scales with a statistically significant difference in scores between baseline and at least one subsequent time point. Blue lines represent mean scores; dotted blue lines represent standard devi-

1 week after the first procedure and returned to baseline at all subsequent time points. However, fatigue also worsened 1 week after the second procedure, and (abdominal) pain worsened 1 week after all three procedures. All worsening PROs (except for appetite loss) were clinically relevant, but all eventually returned to baseline scores 4 weeks after the third procedure. Despite these changes in several PROs, it should also be noted that all other analyzed PROs did not

ations; hollow dots represent statistically significant and clinically relevant differences compared to baseline

change during trial treatment. This is a promising finding in this vulnerable study population (high disease burden, eight patients with signet ring cell differentiation) who generally have a poor prognosis with potentially rapidly deteriorating quality of life.

Thirteen other studies assessed PROs in patients undergoing PIPAC for peritoneal metastases [32-44]. However, six of these specifically focused on PIPAC-cisplatin-doxorubicin 
for non-colorectal primaries (ovarian cancer [32, 33], gastric cancer [34, 35], peritoneal mesothelioma [36], and endometrial and breast cancer [37]. Six other studies [38-43], all focusing on PIPAC with various drugs for various primaries (including oxaliplatin for colorectal cancer), did not report specific outcomes for (e)PIPAC-OX for CPM. Only one of thirteen studies specifically assessed PROs of PIPAC-OX for CPM [44]. However, this study did not report separate PROs of PIPAC-OX monotherapy and PIPAC-OX with concomitant systemic therapy. Altogether, the results of the present study could not be meaningfully compared with the existing literature. Hence, the present study provides detailed insights into PROs during repetitive (e)PIPAC-OX monotherapy for isolated unresectable CPM. Results of the present study may be used to inform patients and physicians about the burden and side effects of ePIPAC-OX in this setting. Several arguments may indicate that the worsening of PROs was most likely related to ePIPAC-OX rather than concomitant treatments or disease progression. First, none of the patients in the present study received concomitant systemic therapy. Second, patients with disease progression did not receive further questionnaires. Third, in all worsening PROs, this worsening was seen at 1 week postoperatively and not at 4 weeks postoperatively. The possibility of treatment-related worsening of PROs should be taken into account by physicians when proposing (e)PIPAC-OX in the palliative setting.

Nevertheless, other treatments for CPM may affect PROs as well. Two studies reported a gradual deterioration of several PROs during treatment with systemic chemotherapy, being the global health status, physical functioning, social functioning, emotional functioning, fatigue, and pain [45, 46]. After cytoreductive surgery and HIPEC, one study reported a gradual deterioration of several PROs (global health status, physical functioning, social functioning, emotional functioning, fatigue, and pain) whereas a second study reported that several PROs deteriorated shortly after surgery (physical well-being, functional well-being, fatigue, pain) but recovered quickly and remained stable during followup [45, 47]. No direct comparisons of PROs during treatment with PIPAC and during treatment with, e.g., systemic chemotherapy were found. Future studies should focus on this comparison to put the reported changes in PROs during treatment with PIPAC-OX into perspective.

Together with a French multicenter retrospective cohort study and the safety and feasibility report of the CRC-PIPAC trial $[15,48]$, the present study suggests that abdominal pain is the most relevant worsening PRO after ePIPAC-OX. Abdominal pain after (e)PIPAC-OX is probably caused by a combination of local pain at trocar sites and diffuse abdominal pain due to chemotherapy-induced chemical peritonitis. This may be drug dependent, as previous reports showed that PIPAC-OX results in a greater inflammatory response and postoperative morphine demand than cisplatin/doxorubicinbased PIPAC [49, 50]. The other worsening gastrointestinal symptoms (i.e., appetite loss, diarrhea, flatulence) may be a result of local chemotherapy-induced changes in gastrointestinal motility. The combination of these worsening symptoms, the effects of general anesthesia, and the (toxicity of) the relevant systemic oxaliplatin uptake after (e)PIPACOX [51, 52] could have led to the observed worsening of more general PROs such as fatigue or physical functioning. The role of electrostatic precipitation and the concomitant intravenous administration of 5-fluorouracil/leucovorin in observed PROs and side effects are currently unknown and may be subject of future research.

While the present study assessed ePIPAC-OX monotherapy, several PIPAC centers regularly offer (e)PIPAC$\mathrm{OX}$ in combination with palliative systemic therapy with the aim to maximize intraperitoneal disease control [13]. Three 6-weekly cycles of first-line systemic chemotherapy and bevacizumab followed by ePIPAC-OX (i.e., bidirectional treatment) are currently investigated in 20 patients with isolated unresectable CPM in an ongoing, multicenter, single-arm phase 2 trial (CRC-PIPAC-II,Netherlands Trial Register: NL8303) [53]. In this trial, PROs (calculated from EQ-5D-5L, EORTC QLQ-C30, and EORTC QLQ-CR29) are explored after the first 6 weeks of first-line palliative systemic therapy (before the first ePIPAC-OX) and 1 and 4 weeks after each ePIPAC-OX procedure. Although the population of CRC-PIPAC-II slightly differs from the population of the present study (which also included patients in later lines of palliative treatment), CRC-PIPAC-II may increase insight in the difference in PROs between bidirectional treatment and ePIPAC-OX monotherapy.

Three other single-arm trials are currently assessing PROs during or after (e)PIPAC-OX for unresectable CPM. A Singaporean single-center phase 1 trial assesses EORTC QLQ-C30 at 6 and 12 weeks during 6-weekly PIPAC-OX monotherapy in five dose levels $\left(45 \mathrm{mg} / \mathrm{m}^{2}\right.$ to $\left.150 \mathrm{mg} / \mathrm{m}^{2}\right)$ in patients with unresectable peritoneal metastases of various 

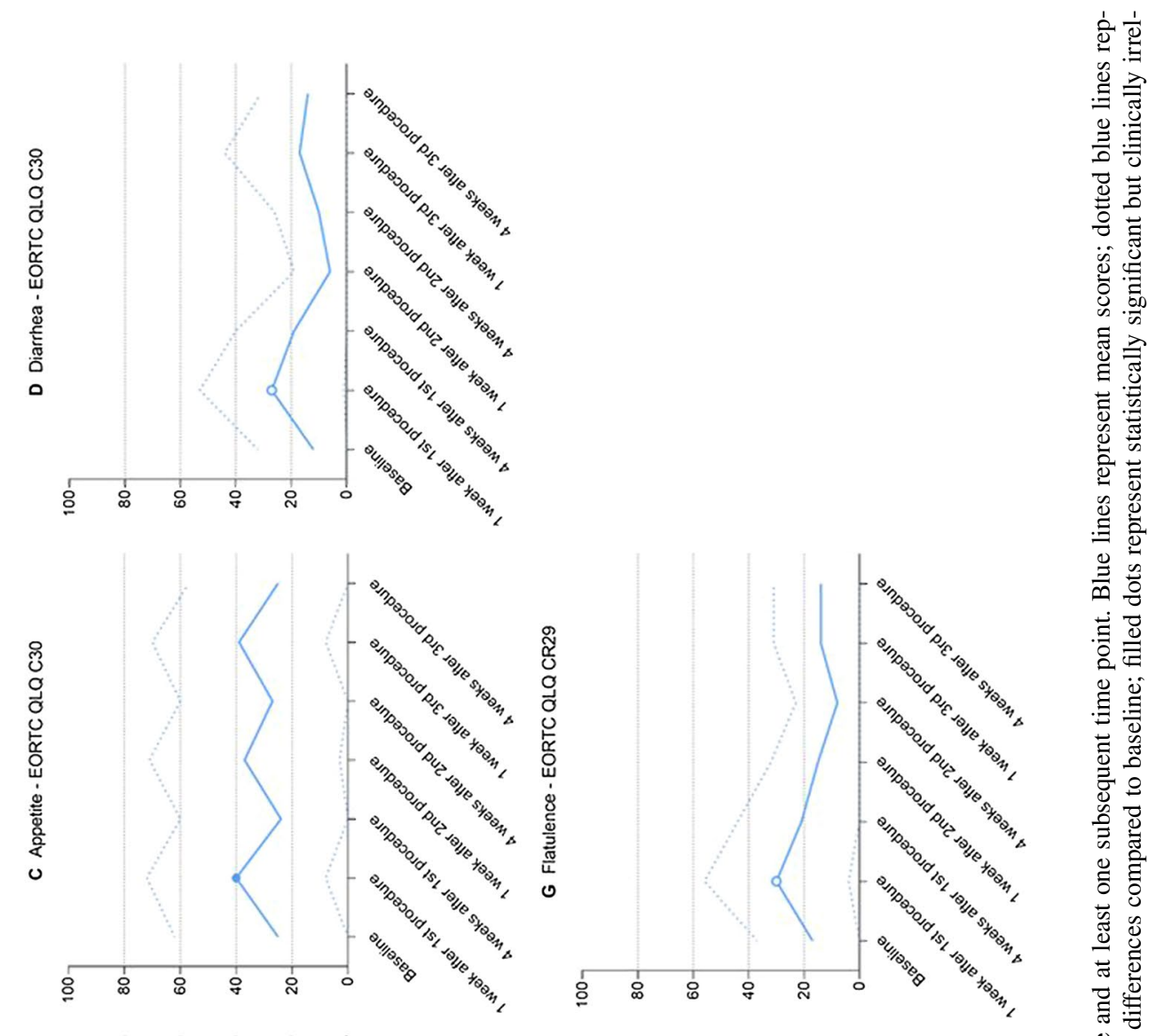

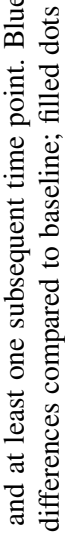
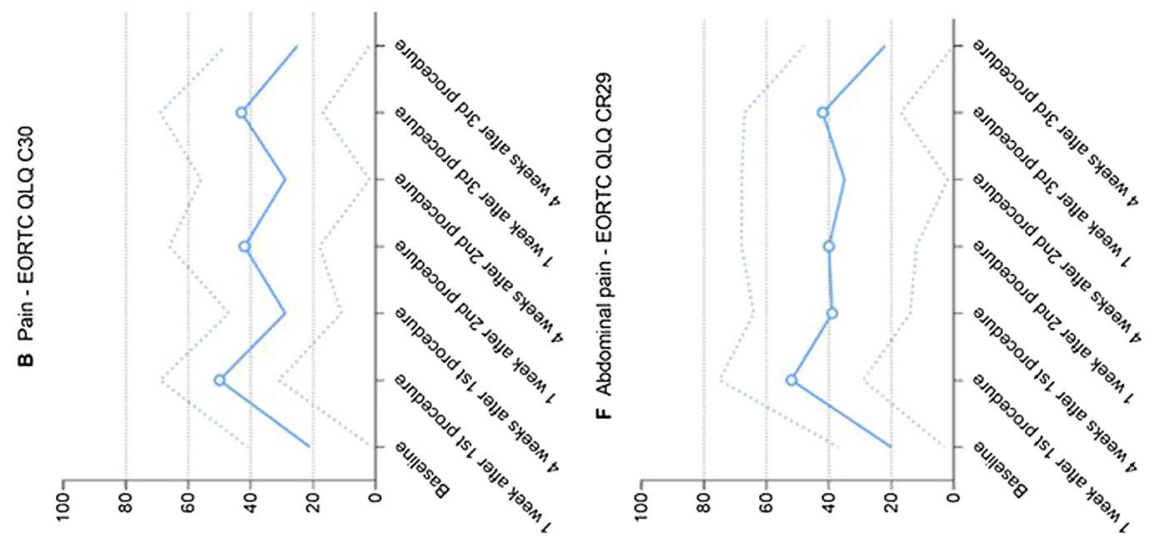

离

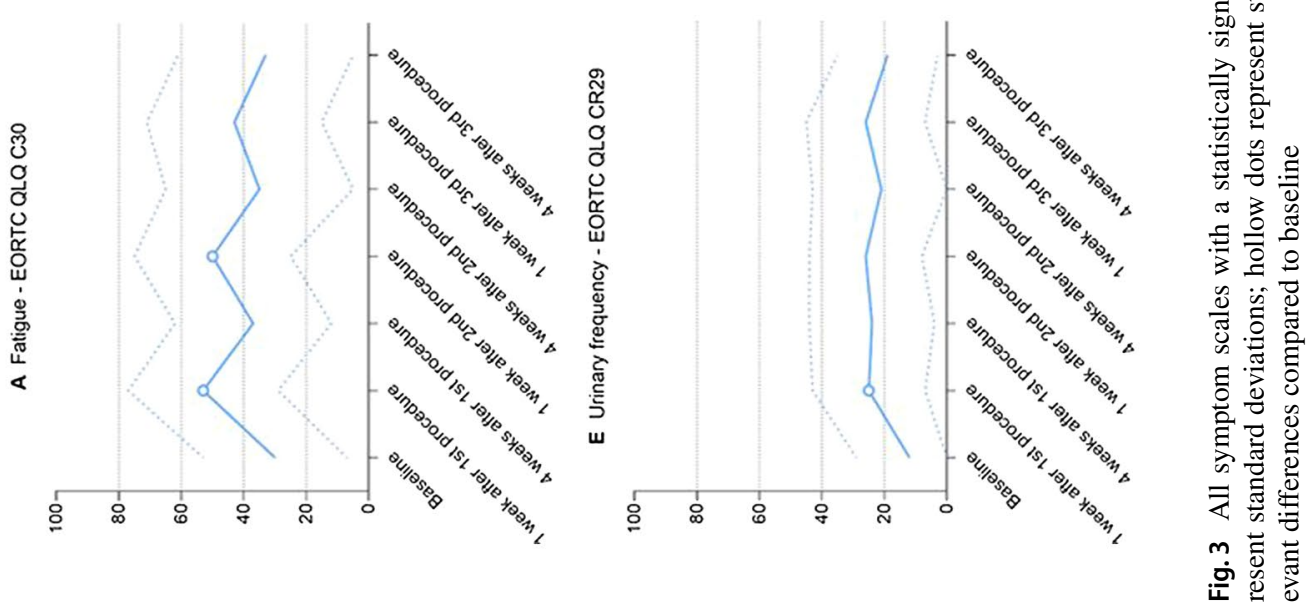


origins (including colorectal) who completed, refused, or were unable to tolerate first-line systemic therapy (Clinicaltrials.gov: NCT03172416) [54]. A British single-center phase 2 trial analyzes EORTC QLQ-C30 just before every procedure during 6-to-8-week PIPAC-OX, with or without concomitant systemic chemotherapy therapy, in 30 patients with unresectable CPM in various lines of palliative treatment (Clinicaltrials.gov: NCT03868228). A Danish singlecenter phase 2 trial assesses EORTC QLQ-C30 at 4 months during (or after) (e)PIPAC with various drugs (including oxaliplatin), with or without concomitant palliative systemic therapy, in patients with unresectable peritoneal metastases of various origins (including cpm) in various lines of palliative treatment (PIPAC-OPC2,Clinicaltrials. gov: NCT03287375) [55]. The international PIPAC registry (Clinicaltrials.gov: NCT03210298), which also analyzes EORTC QLQ-C30, may provide further insight in real-world PROs outside of clinical trials.

The results of the CRC-PIPAC trial, ongoing trials, and the international PIPAC registry may be used to design future randomized trials to determine the role of (e)PIPAC$\mathrm{OX}$ in the treatment of patients with isolated (initially) unresectable CPM. Importantly, international consensus must be reached on the most appropriate interventions (e.g., PIPAC-OX monotherapy, bidirectional treatment), settings (e.g., neoadjuvant, first-line palliative, refractory), frameworks (e.g., superiority, equivalence, non-inferiority), and endpoints (e.g., survival, PROs, combination of both) of such trials. If PROs will be used as a primary endpoint, the results of the present study and ongoing trials may be used to choose the most appropriate PROs and may serve as a basis for sample size calculations.

The small sample size was the main limitation of this explorative study. A larger sample size may have detected statistically significant fluctuations in PROs that could have been clinically relevant. Furthermore, while questionnaire response rates were high, trial treatment was stopped before the second procedure in four of twenty patients, and before the third procedure in an additional three patients. Although inevitable in trials including patients with a very poor prognosis, this drop-out reduced the statistical power of comparisons of baseline scores with scores after the second and third procedures. Nevertheless, despite the small sample size, linear mixed modeling analyses allowed for the detection of both statistically significant and clinically relevant findings.

\section{Conclusions}

Patients with isolated unresectable CPM receiving repetitive ePIPAC-OX monotherapy (i.e., without palliative systemic therapy in between subsequent procedures) had clinically relevant but reversible worsening of several PROs during trial treatment, mainly after the first procedure. All worsening PROs eventually returned to baseline 4 weeks after the third procedure. Despite these changes in several PROs, it should also be noted that all other analyzed PROs did not change during trial treatment. The results of the present study may be used to inform patients about the burden of (e)PIPAC-OX, should be taken into account by physicians when proposing (e)PIPAC-OX in the palliative setting, and could help designing future PRO-focused randomized trials to determine the role of (e)PIPAC-OX in the palliative treatment of CPM.

Supplementary Information The online version contains supplementary material available at https://doi.org/10.1007/s00464-021-08802-6.

\section{Acknowledgements None.}

Funding This work was supported by the Catharina Research Foundation (Grant Number: 2017-5) and the St. Antonius Research Foundation (Grant Number: 17.4). The funders had no role in the study design, in the collection, analysis and interpretation of data, in the writing of the report, and in the decision to submit the article for publication.

\section{Declarations}

Disclosures Robin J. Lurvink, Koen P. Rovers, Emma C.E. Wassenaar, Checca Bakkers, Jacobus W.A. Burger, Geert-Jan M. Creemers, Maartje Los, Floortje Mols, Marinus J. Wiezer, Simon W. Nienhuijs, and Djamila Boerma have no conflicts of interest or financial ties to disclose. Ignace H.J.T. de Hingh: an unrestricted research grant outside the submitted work from RanD/QPS and Roche, paid to the institute.

Open Access This article is licensed under a Creative Commons Attribution 4.0 International License, which permits use, sharing, adaptation, distribution and reproduction in any medium or format, as long as you give appropriate credit to the original author(s) and the source, provide a link to the Creative Commons licence, and indicate if changes were made. The images or other third party material in this article are included in the article's Creative Commons licence, unless indicated otherwise in a credit line to the material. If material is not included in the article's Creative Commons licence and your intended use is not permitted by statutory regulation or exceeds the permitted use, you will need to obtain permission directly from the copyright holder. To view a copy of this licence, visit http://creativecommons.org/licenses/by/4.0/.

\section{References}

1. van der Geest LG, Lam-Boer J, Koopman M et al (2015) Nationwide trends in incidence, treatment, and survival of colorectal cancer patients with synchronous metastases. Clin Exp Metastasis 32:457-465

2. Lurvink RJ, Bakkers C, Rijken A et al (2020) Increase in the incidence of synchronous and metachronous peritoneal metastases in patients with colorectal cancer: a nationwide study. Eur J Surg Oncol S0748-7983(20):31031-31033

3. Razenberg LG, Lemmens VE, Verwaal VJ et al (2016) Challenging the dogma of colorectal peritoneal metastases as an 
untreatable condition: results of a population-based study. Eur J Cancer 65:113-120

4. Quere P, Facy O, Manfredi S et al (2015) Epidemiology, management, and survival of peritoneal carcinomatosis from colorectal cancer: a population-based study. Dis Colon Rectum 58:743-752

5. Dedrick RL, Myers CE, Bungay PM, DeVita VT Jr (1978) Pharmacokinetic rationale for peritoneal drug administration in the treatment of ovarian cancer. Cancer Treat Rep 62:1-11

6. Dedrick RL, Flessner MF (1997) Pharmacokinetic problems in peritoneal drug administration: tissue penetration and surface exposure. J Natl Cancer Inst 89:480-487

7. Markman M (2003) Intraperitoneal antineoplastic drug delivery: rationale and results. Lancet Oncol 4(5):277-283

8. Solass W, Hetzel A, Nadiradze G, Sagynaliev E, Reymond MA (2012) Description of a novel approach for intraperitoneal drug delivery and the related device. Surg Endosc 26:1849-1855

9. Solass W, Herbette A, Schwarz T et al (2012) Therapeutic approach of human peritoneal carcinomatosis with Dbait in combination with capnoperitoneum: proof of concept. Surg Endosc $26: 847-852$

10. Blanco A, Giger-Pabst U, Solass W, Zieren J, Reymond MA (2013) Renal land hepatic toxicities after pressurized intraperitoneal aerosol chemotherapy (PIPAC). Ann Surg Oncol 20:2311-2316

11. Solass W, Kerb R, Mürdter T et al (2014) Intraperitoneal chemotherapy of peritoneal carcinomatosis using pressurized aerosol as an alternative to liquid solution: first evidence for efficacy. Ann Surg Oncol 21:553-559

12. Alyami M, Hübner M, Grass F et al (2019) Pressurised intraperitoneal aerosol chemotherapy: rationale, evidence, and potential indications. Lancet Oncol 20:e368-e377

13. Sgarbura O, Villeneuve L, Alyami M et al (2021) Current practice of pressurized intraperitoneal aerosol chemotherapy (PIPAC): still standardized or on the verge of diversification? Eur J Surg Oncol 47(1):149-156

14. Rovers KP, Lurvink RJ, Wassenaar EC et al (2019) Repetitive electrostatic pressurised intraperitoneal aerosol chemotherapy (ePIPAC) with oxaliplatin as a palliative monotherapy for isolated unresectable colorectal peritoneal metastases: protocol of a Dutch, multicentre, open-label, single-arm, phase II study (CRC-PIPAC). BMJ Open 9(7):e030408

15. Rovers KP, Wassenaar EC, Lurvink RJ et al (2021) Pressurized Intraperitoneal Aerosol Chemotherapy (oxaliplatin) for unresectable colorectal peritoneal metastases: a multicenter, single-arm, phase II trial (CRC-PIPAC). Ann Surg Oncol. https://doi.org/10. 1245/s10434-020-09558-4

16. Elias D, Bonnay M, Puizillou JM et al (2002) Heated intra-operative intraperitoneal oxaliplatin after complete resection of peritoneal carcinomatosis: pharmacokinetics and tissue distribution. Ann Oncol 13(2):267-272

17. Giacchetti S, Perpoint B, Zidani R et al (2000) Phase III multicenter randomized trial of oxaliplatin added to chronomodulated fluorouracil-leucovorin as first-line treatment of metastatic colorectal cancer. J Clin Oncol 18:136-147

18. Reymond M, Demtröder C, Solass W, Winnekendonk G, Tempfer C (2016) Electrostatic precipitation pressurized intraperitoneal aerosol chemotherapy (ePIPAC): first in-human application. Pleura Peritoneum 1(2):109-116

19. Kakchekeeva T, Demtröder C, Herath NI et al (2016) In vivo feasibility of electrostatic precipitation as an adjunct to pressurized intraperitoneal aerosol chemotherapy (ePIPAC). Ann Surg Oncol 23(Suppl 5):592-598

20. Eisenhauer EA, Therasse P, Bogaerts J et al (2009) New response evaluation criteria in solid tumours: revised RECIST guideline (version 1.1). Eur J Cancer 45:228-247
21. Herdman M, Gudex C, Lloyd A et al (2011) Development and preliminary testing of the new five-level version of EQ-5D (EQ5D-5L). Qual Life Res 20:1727-1736

22. Aaronson NK, Ahmedzai S, Bergman B et al (1993) The European Organization for Research and Treatment of Cancer QLQ-C30: a quality-of-life instrument for use in international clinical trials in oncology. J Natl Cancer Inst 85:365-376

23. Stiggelbout AM, Kunneman M, Baas-Thijssen MC et al (2016) The EORTC QLQ-CR29 quality of life questionnaire for colorectal cancer: validation of the Dutch version. Qual Life Res 25:1853-1858

24. EuroQol Research Foundation. EQ-5D-5L User Guide, 2019.

25. Fayers PM, Aaronson NK, Bjordal K, Groenvold M, Curran D, Bottomley A; on behalf of the EORTC Quality of Life Group. The EORTC QLQ-C30 Scoring Manual (3rd Edition). European Organisation for Research and Treatment of Cancer, Brussels, 2001.

26. Whistance RN, Conroy T, Chie W et al (2009) Clinical and psychometric validation of the EORTC QLQ-CR29 questionnaire module to assess health-related quality of life. Eur J Cancer 45:3017-3026

27. van Hout $B$, Janssen MF, Feng YS et al (2012) Interim scoring for the EQ-5D-5L: mapping the EQ-5D-5L to EQ-5D-3L value sets. Value Health 15:708-715

28. Norman GR, Sloan JA, Wyrwich KW (2003) Interpretation of changes in health-related quality of life: the remarkable universality of half a standard deviation. Med Care 41:582-592

29. Cocks K, King MT, Velikova G et al (2012) Evidence-based guidelines for interpreting change scores for the European organisation for the research and treatment of cancer quality of life questionnaire core 30. Eur J Cancer 48(11):1713-1721

30. Osoba D, Rodrigues G, Myles J, Zee B, Pater J (1998) Interpreting the significance of changes in health-related quality of life scores. J Clin Oncol 16(1):139-144

31. Pickard AS, Neary MP, Cella D (2007) Estimation of minimally important differences in EQ-5D utility and VAS scores in cancer. Health Qual Life Outcomes 5:70

32. Tempfer CB, Winnekendonk G, Solass W et al (2015) Pressurized intraperitoneal aerosol chemotherapy in women with recurrent ovarian cancer: a phase 2 study. Gynecol Oncol 137:223-228

33. Tempfer CB, Rezniczek GA, Ende P, Solass W, Reymond MA (2015) Pressurized intraperitoneal aerosol chemotherapy with cisplatin and doxorubicin in women with peritoneal carcinomatosis: a cohort study. Anticancer Res 35:6723-6729

34. Gockel I, Jansen-Winkeln B, Haase L et al (2018) Pressurized intraperitoneal aerosol chemotherapy (PIPAC) in gastric cancer patients with peritoneal metastasis (PM): results of a single-center experience and register study. J Gastric Cancer 18:379-391

35. Struller F, Horvath P, Solass W et al (2019) Pressurized intraperitoneal aerosol chemotherapy with low-dose cisplatin and doxorubicin (PIPAC C/D) in patients with gastric cancer and peritoneal metastasis: a phase II study. Ther Adv Med Oncol 11:1758835919846402

36. Giger-Pabst U, Demtröder C, Falkenstein TA et al (2018) Pressurized intraperitoneal aerosol chemotherapy (PIPAC) for the treatment of malignant mesothelioma. BMC Cancer 18:442

37. Rezniczek GA, Giger-Pabst U, Thaher O et al (2020) Pressurized intraperitoneal aerosol chemotherapy (PIPAC) for rare gynecologic indications: peritoneal metastases from breast and endometrial cancer. BMC Cancer 20:1122

38. Odendahl K, Solass W, Demtröder C et al (2015) Quality of life of patients with end-stage peritoneal metastasis treated with pressurized intraperitoneal aerosol chemotherapy (PIPAC). Eur J Surg Oncol 41:1379-1385

39. Robella M, Vaira M, de Simone M (2016) Safety and feasibility of pressurized intraperitoneal aerosol chemotherapy (PIPAC) 
associated with systemic chemotherapy: an innovative approach to treat peritoneal carcinomatosis. World J Surg Oncol 14:128

40. Teixeira Farinha H, Grass F, Kefleyesus A et al (2017) Impact of pressurized intraperitoneal aerosol chemotherapy on quality of life and symptoms in patients with peritoneal carcinomatosis: a retrospective cohort study. Gastroenterol Res Pract 2017:4596176

41. Graversen M, Detlefsen S, Bjerregaard JK, Fristrup CW, Pfeiffer P, Mortensen MB (2018) Prospective, single-center implementation and response evaluation of pressurized intraperitoneal aerosol chemotherapy (PIPAC) for peritoneal metastasis. Ther Adv Med Oncol 10:1758835918777036

42. de Simone M, Vaira M, Argenziano M et al (2020) Pressurized intraperitoneal aerosol chemotherapy (PIPAC) with oxaliplatin, cisplatin, and doxorubicin in patients with peritoneal carcinomatosis: an open-label, single-arm, phase II clinical trial. Biomedicines $8: 102$

43. Taibi A, Teixeira Farinha H, Durand Fontanier S, Sayedalamin Z, Hübner M, Sgarbura O (2020) Pressurized intraperitoneal aerosol chemotherapy enhanced by electrostatic precipitation (ePIPAC) for patients with peritoneal metastases. Ann Surg Oncol. https:// doi.org/10.1245/s10434-020-093320-6

44. Tabchouri N, Buggisch J, Demtroder CR et al (2021) Pressurized intraperitoneal aerosol chemotherapy for colorectal peritoneal metastases. Ann Surg Oncol. https://doi.org/10.1245/s/ 10434-020-09508-0

45. Cashin PH, Mahteme H, Syk I, Frödin JE, Glimelius B, Graf W (2018) Quality of life and cost effectiveness in a randomized trial of patients with colorectal cancer and peritoneal metastases. Eur J Surg Oncol 44(7):983-990

46. Andre T, Amonkar M, Norquist JM et al (2021) Health-related quality of life in patients with microsatellite instability-high or mismatch repair deficient metastatic colorectal cancer treated with first-line pembrolizumab versus chemotherapy (Keynote-177): an open-label, randomised, phase 3 trial. Lancet Oncol 22(5):665-677

47. Moaven O, Votanopoulos KI, Shen P et al (2020) Health-related quality of life after cytoreductive surgery/HIPEC for mucinous appendiceal cancer: results of a multicenter randomized trial comparing oxaliplatin and mitomycin. Ann Surg Oncol 27(3):772-780
48. Sgarbura O, Hübner M, Alyami M et al (2019) Oxaliplatin use in pressurized intraperitoneal aerosol chemotherapy (PIPAC) is safe and effective: a multicenter study. Eur J Surg Oncol 45(12):2386-2391

49. Teixeira Farinha H, Grass F, Labga I, Pache B, Demartines N, Hübner M (2018) Inflammatory response and toxicity after pressurized intraperitoneal aerosol chemotherapy. J Cancer 9(1):13-20

50. Graversen M, Lundell L, Fristrup C, Pfeiffer P, Mortensen MB (2018) Pressurized intraperitoneal aerosol chemotherapy (PIPAC) as an outpatient procedure. Pleura Peritoneum 3(4):20180128

51. Lurvink RJ, Tajzai R, Rovers KP et al (2021) Systemic pharmacokinetics of oxaliplatin after intraperitoneal administration by electrostatic pressurized intraperitoneal aerosol chemotherapy (ePIPAC) in patients with unresectable colorectal peritoneal metastases in the CRC-PIPAC trial. Ann Surg Oncol 28:265-272

52. Dumont F, Passot C, Raoul JL et al (2020) A phase I dose-escalation study of oxaliplatin delivered via a laparosocpic approach using pressurised intraperitoneal aerosol chemotherapy for advanced peirtoneal metastases of gastrointestintal tract cancers. Eur J Cancer 140:37-44

53. Lurvink RJ, Rauwerdink P, Rovers KP et al (2021) First-line palliative systemic therapy alternated with electrostatic pressurised intraperitoneal aerosol chemotherapy (oxaliplatin) for isolated unresectable colorectal peritoneal metastases: protocol of a multicentre, single-arm, phasde II study (CRC-PIPAC-II). BMJ Open 11:e044811

54. Kim G, Tan HL, Chen E et al (2018) Study protocol: phase 1 dose escalating study of pressurized intra-peritoneal aerosol chemotherapy (PIPAC) with oxaliplatin in peritoneal metastasis. Pleura Peritoneum 3:20180118

55. Graversen M, Detlefsen S, Asmussen J et al (2018) Treatment of peritoneal carcinomatosis with pressurized intraperitoneal aerosol chemotherapy: PIPAC-OPC2. Pleura Peritoneum 3:20180108

Publisher's Note Springer Nature remains neutral with regard to jurisdictional claims in published maps and institutional affiliations.

\section{Authors and Affiliations}

\section{Robin J. Lurvink ${ }^{1,2} \oplus$ - Koen P. Rovers ${ }^{1} \cdot$ Emma C. E. Wassenaar $^{3} \cdot$ Checca Bakkers $^{1} \cdot$ Jacobus W. A. Burger ${ }^{1}$. Geert-Jan M. Creemers ${ }^{4} \cdot$ Maartje Los ${ }^{5} \cdot$ Floortje Mols $^{2,6} \cdot$ Marinus J. Wiezer $^{3}$. Simon W. Nienhuijs ${ }^{1}$. Djamila Boerma ${ }^{3} \cdot$ Ignace H. J. T. de Hingh ${ }^{1,2,7}$}

1 Department of Surgery, Catharina Cancer Institute, PO Box 1350, 5602 ZA Eindhoven, The Netherlands

2 Department of Research and Development, Netherlands Comprehensive Cancer Organization, Utrecht, The Netherlands

3 Department of Surgery, St. Antonius Hospital, PO Box 2500, 3430 EM Nieuwegein, The Netherlands

4 Department of Medical Oncology, Catharina Cancer Institute, PO Box 1350, 5602 ZA Eindhoven, The Netherlands
5 Department of Medical Oncology, St. Antonius Hospital, PO Box 2500, 3430 EM Nieuwegein, The Netherlands

6 Center of Research on Psychology in Somatic Disorders, Department of Medical and Clinical Psychology, Tilburg University, PO Box 90153, 5000 LE Tilburg, The Netherlands

7 GROW - School for Oncology and Developmental Biology, Maastricht University, PO Box 616, 6200 MD Maastricht, The Netherlands 